\title{
SMC-mediated chromosome mechanics: a conserved scheme from bacteria to vertebrates?
}

\author{
Tatsuya Hirano ${ }^{1}$ \\ Cold Spring Harbor Laboratory, Cold Spring Harbor, New York 11724 USA
}

The assembly of mitotic chromosomes is a fundamental cellular event that ensures the faithful segregation of genetic information during cell division. It involves two processes that are, in principle, mechanistically distinct. The first process, establishment of sister chromatid cohesion, occurs during or soon after chromosome duplication. The linkage between two sister chromatids is maintained throughout $\mathrm{G}_{2}$ phase and secures the bipolar attachment of each chromosome to the spindle apparatus from prometaphase through metaphase. The second process, chromosome condensation, can be visualized most dramatically at the onset of mitosis, converting the chromatids into physically strong, rod-shaped structures. The resulting metaphase chromosome consists of two condensed sister chromatids tightly paired at their centromeric regions as well as along their entire arms. At the onset of anaphase, the linkage between the sister chromatids is dissolved, allowing them to be pulled apart to opposite poles of the cell. This dynamic behavior of chromosomes had been described by cytologists long before the central role of DNA as the genetic material was recognized and long before the biochemical basis of cell cycle progression was elucidated. Nevertheless, the molecular mechanisms underlying these structural changes of chromosomes have remained poorly understood to date. A recent breakthrough in this field was the discovery of a novel family of chromosomal ATPases, the structural maintenance of chromosome (SMC) family. Genetic and biochemical studies have shown that in eukaryotes, two distinct classes of SMC protein complexes, condensins and cohesins, play central roles in chromosome condensation and sister-chromatid cohesion, respectively. Other SMC protein complexes are involved in chromosome-wide gene repression /dosage compensation) and recombinational repair. SMC proteins are also conserved among bacteria and archaea, and their functional characterization is just beginning to emerge. In this paper recent progress in the SMC field is discussed and an attempt is made to deduce a basic scheme of SMC-mediated chromosome mechanics that might be common from bacteria to vertebrates. For recent reviews

1E-MAIL hirano@cshl.org; FAX (516) 367-8815. on this topic, see Koshland and Strunnikov (1996), Hirano (1998) and Jessberger et al. (1998).

\section{Evolutionary conservation of SMC proteins}

SMC proteins are large polypeptides (between 1000 and 1500 amino acids long) that share common structural motifs, including an amino-terminal nucleotide-binding motif (the Walker A motif), two central coiled-coil motifs, and a carboxy-terminal conserved sequence, termed the DA-box (perhaps related to the Walker B motif). Most eukaryotes have multiple SMC proteins, which have been classified into four subtypes (from SMC1- to SMC4type proteins; Tables 1 and 2). Biochemical studies have revealed that SMC proteins form two different types of heterodimers with a remarkable specificity: one combination is SMC1 and SMC3, and the other is SMC2 and SMC4 (Hirano and Mitchison 1994; Jessberger et al. 1996; Sutani and Yanagida 1997; Lieb et al. 1998; Losada et al. 1998; Schmiesing et al. 1998). The SMC1-SMC3 and SMC2-SMC4 heterodimers further associate with different sets of non-SMC subunits, producing a variety of large protein complexes with diverse functions (Fig. 1; Tables 1 and 2). smc genes are also found in most of the bacterial and archaeal genomes. Each of the genomes contains a single $s m c$ gene, suggesting that bacterial or archaeal SMC proteins might function as homodimers. This idea has been confirmed recently by biochemical studies of the Bacillus subtilis SMC protein (Hirano and Hirano 1998; Melby et al. 1998). For an updated version of phylogenetic trees of SMC proteins, refer to Melby et al. (1998).

\section{Condensins and chromosome condensation}

\section{Components}

SMC2- and SMC4-type proteins act as core components of condensins, chromosome condensation protein complexes originally purified from Xenopus laevis egg extracts (Hirano and Mitchison 1994; Hirano et al. 1997). The egg extracts contain two forms of the condensin complexes with sedimentation coefficients of $8 \mathrm{~S}$ and 
Table 1. Protein complexes containing SMC2-SMC4

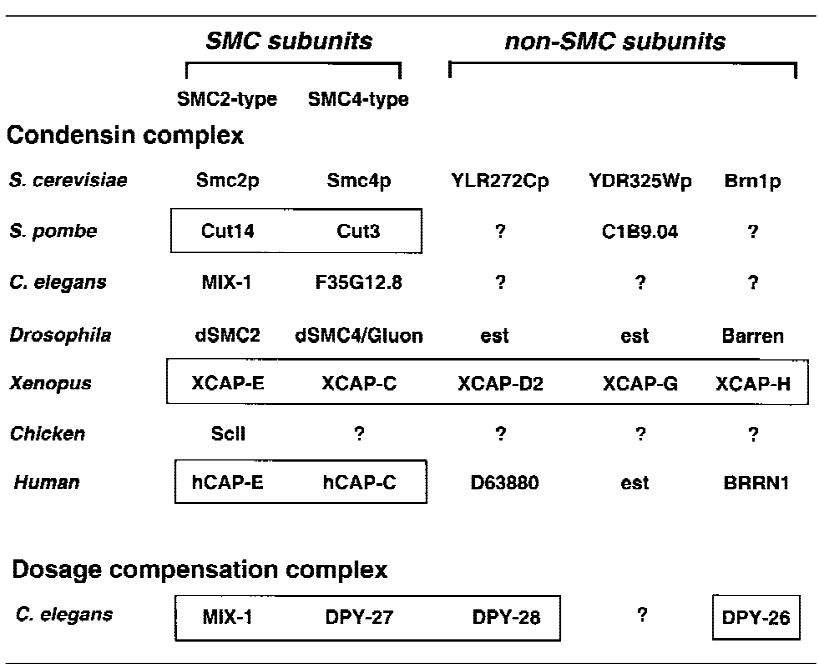

Eukaryotic sequences homologous to the subunits of the Xenopus 13S condensin are shown. Proteins are enclosed by rectangles when they have been shown experimentally to interact with each other. Brnlp (I. Ouspenski, pers. comm.); dSMC2 and dSMC4 (X. Shen and C. Wu, pers. comm.); Gluon (H. Bellen, pers. comm.); XCAP-G (T. Hirano; unpubl.); hCAP-E and hCAP-C (Schmiesing et al. 1998); BRRN1 (Cabello et al. 1997); DPY-28 (B. Meyer, pers. comm.). (est) Partial homologous sequences are deposited in the EST databases. Other references are in the text.

13S. The $8 \mathrm{~S}$ form (8S condensin) is a heterodimer of XCAP-E (SMC2-type) and XCAP-C (SMC4-type), whereas the $13 \mathrm{~S}$ form (13S condensin) contains three additional subunits, XCAP-D2, XCAP-G, and XCAP-H (Fig. 1; Table 1). As judged by immunodepletion and addback experiments, $13 \mathrm{~S}$ condensin is the active complex required for chromosome condensation in this cell-free system. The functional role of $8 \mathrm{~S}$ condensin is unclear. In a cell lysate of Schizosaccharomyces pombe, a $~ 13 S$ complex is detected whose subunit composition is similar to that of the Xenopus 13S condensin complex: It contains Cut14 (SMC2-type), Cut3 (SMC4-type) (Sutani and Yanagida 1997), and three other subunits (T. Sutani and M. Yanagida, pers. comm.). Mutations in cut3 or cut14 lead to defects in chromosome condensation and segregation in S. pombe (Saka et al. 1994). An smc2 mutant in Saccharomyces cerevisiae also shows similar phenotypes (Strunnikov et al. 1995). These genetic studies in yeasts demonstrate that SMC2- and SMC4-type proteins are required for chromosome condensation in vivo and further support the idea that condensation is a prerequisite for successful segregation of chromosomes.

Amino acid sequence analysis of XCAP-D2, XCAP-G, and XCAP-H shows that they are all highly conserved from yeast to humans (Table 1; Hirano et al. 1997; Kimura et al. 1998; T. Hirano, unpubl.). XCAP-H is homologous to a Drosophila melanogaster protein called Barren (Bhat et al. 1996). Mutants in barren are defective in chromosome segregation in early embryos in Drosophila, consistent with its role in mitotic chromosome dynamics. More recently, it has been shown that Brnlp, the yeast homolog of Barren/XCAP-H, is an essential nuclear protein required for condensation of rDNA (I. Ouspenski, pers. comm.). In Drosophila, Barren was shown to interact with topoisomerase II and to modulate its enzymatic activity (Bhat et al. 1996). However, no such interaction has been detected in Xenopus, at least before the two proteins are targeted to chromosomes (Hirano et al. 1997). Further studies will be required to clarify the functional interactions between condensin subunits and topoisomerase II. Whereas the primary structures of these non-SMC subunits provide little information about their biochemical functions, a recent study from the Xenopus cell-free system suggests that they play regulatory roles in condensin functions (see below). Genetic studies of the XCAP-D2 and XCAP-G homologs are yet to be described.

\section{Mechanism}

How does the condensin complex interact with chromatin and induce its condensation? Although we are still in a stage too early to answer this mechanistic question, the first clue was provided by the finding that $13 \mathrm{~S}$ condensin can induce superhelical tension into DNA by utilizing the energy of ATP hydrolysis (Kimura and Hirano 1997). Although 13S condensin has no topoisomerase activity, it can introduce positive supercoils into relaxed circular DNA in the presence of topoisomerase I. This supercoiling reaction is ATP dependent and requires a stoichiometric amount of $13 \mathrm{~S}$ condensin. $13 \mathrm{~S}$ condensin has a DNA-stimulated ATPase activity, consistent with

Table 2. Protein complexes containing SMC1-SMC3

\begin{tabular}{|c|c|c|c|c|c|}
\hline & \multicolumn{2}{|c|}{ SMC subunits } & \multicolumn{3}{|c|}{ non-SMC subunits } \\
\hline Cohesin co & $\begin{array}{l}\text { SMC1-type } \\
\text { nplex }\end{array}$ & smc3-type & & & \\
\hline S. cerevisiae & Smc1p & Smc3p & Mcd1p/Scc1p & $?$ & $?$ \\
\hline S. pombe & Psm1 & Psm3 & Rad21 & $?$ & $?$ \\
\hline A. nidulans & $?$ & SudA & $?$ & $?$ & $?$ \\
\hline C. elegans & F28B3.7 & Y47D3 & F10G7.4/K08A8.3 & $?$ & $?$ \\
\hline Drosophila & $?$ & DCAP & est & $?$ & $?$ \\
\hline Xenopus & XSMC1 & XSMC3 & XRAD21 & p155 & p95 \\
\hline Muman & hSMC1/Sb1.8 & hSMC3/HCAP & hHR21 & $?$ & $?$ \\
\hline \multicolumn{6}{|l|}{$\mathrm{RC}-1$} \\
\hline Bovine & bSMC1 & bSMC3 & DNA pol $\varepsilon$ & ligase III & \\
\hline
\end{tabular}

Eukaryotic sequences homologous to the subunits of the Xenopus 14S cohesin complex are shown. The identity of p155 and p95 is currently unknown. Proteins are enclosed by rectangles when they have been shown experimentally to interact with each other. Psm1 and Psm3 (T. Sutani, T. Tomonaga, and M. Yanagida, pers. comm.); DCAP (Hong and Ganetzky 1996); hSMC1 and hSMC3 (Schmiesing et al. 1998); Sb1.8 (Rocques et al. 1995); HCAP (Shimizu et al. 1998); hHR21 (McKay et al. 1996). (est) Partial homologous sequences are deposited in the EST databases. Other references are in the text. 
SMC2-SMC4-type

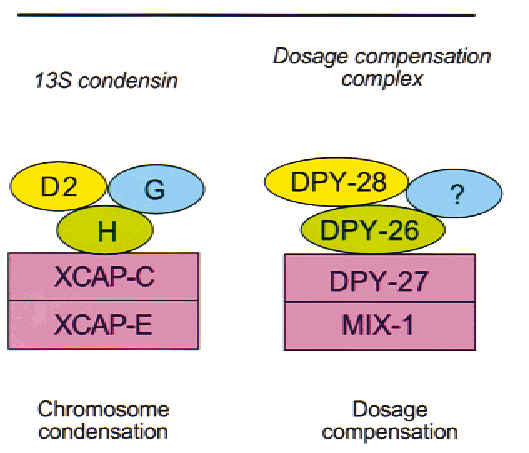

\section{SMC1-SMC3-type}

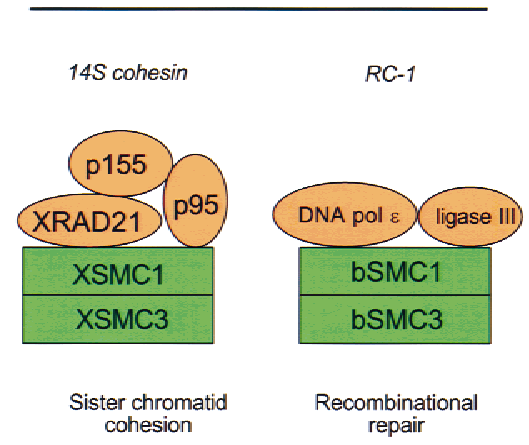

Figure 1. Structural organization of eukaryotic SMC protein complexes. (SMC2-SMC4type) $13 \mathrm{~S}$ condensin is a five-subunit protein complex that plays a central role in chromosome condensation in Xenopus egg cell-free extracts. Two SMC subunits, XCAP-C (SMC4type) and XCAP-E (SMC2-type), constitute the core of this complex. XCAP-H is homologous to the Drosophila Barren protein. The dosage compensation complex has been identified through genetic and biochemical studies in $C$. elegans. It is composed of at least four subunits, including MIX-1 (SMC2-type), DPY-27 (SMC4-variant type), DPY-26 (weakly homologous to XCAP-H), and DPY-28 (homologous to

XCAP-D2). (SMC1-SMC3-type) The 14S cohesin complex, which was originally inferred from genetic studies in yeast and subsequently purified from Xenopus egg extracts, is involved in sister-chromatid cohesion. It contains XSMC1 (SMC1-type), XSMC3 (SMC3-type), and XRAD21 (the Xenopus homolog of S. cerevisiae Scc1p/Mcd1p and S. pombe Rad21). The stoichiometry of each subunit in the complex and the identities of the remaining subunits (p155 and p95) are currently unknown. RC-1 is a recombination protein complex purified from calf thymus that contains DNA polymerase $\epsilon$, ligase III, and two SMC (SMC1- and SMC3-type) subunits. SMC subunits are shown by rectangles; non-SMC subunits are shown by ellipses.

the finding that all SMC proteins share ATP-binding motifs. Moreover, a recent study has shown that the supercoiling activity is regulated during the cell cycle (Kimura et al. 1998). Whereas the interphase and mitotic forms of $13 \mathrm{~S}$ condensin have the same subunit composition, the three non-SMC subunits, XCAP-D2, XCAP-G, and $\mathrm{XCAP}-\mathrm{H}$, are phosphorylated in a mitosis-specific manner. The interphase complex does not support supercoiling and phosphatase treatment of the mitotic complex abolishes its activity, indicating that the supercoiling activity is regulated by mitosis-specific phosphorylation. In vitro phosphorylation and phosphopeptide mapping experiments have shown that cdc2 is likely to be the physiological kinase that phosphorylates and activates 13S condensin. Multiple cdc2 phosphorylation sites are clustered in the carboxy-terminal domain of XCAP-D2 and the amino-terminal domain of XCAP-H. These results reveal a direct functional link between the master mitotic kinase cdc2 and chromosome condensation. They also provide additional evidence that the ATP-dependent supercoiling activity of $13 \mathrm{~S}$ condensin may be physiologically relevant and contribute directly to mitosis-specific condensation.

The second activity found associated with condensin subunits is reannealing of complementary DNA strands. This activity is supported by the Cut3-Cut14 heterodimer of S. pombe (an equivalent of Xenopus $8 \mathrm{~S}$ condensin; Sutani and Yanagida 1997). A combined biochemical and genetic analysis suggests a strong correlation between the reannealing activity in vitro and the condensation activity in vivo. It should be emphasized, however, that the reannealing reaction does not require ATP and is considered to be only a part of the condensin activity (Yanagida 1998). To better understand these biochemical activities at a mechanistic level, functional dissection of the condensin complex as well as of its individual subunits is essential. Such analysis has just been initiated with the yeast SMC proteins (Akhmedov et al. 1998). A major DNA-binding activity appears to reside in the car- boxy-terminal domain of Smc2p (and Smclp), and this domain alone is able to support a partial reannealing activity. The latter observation is somehow inconsistent with a previous work showing that heterodimeric association of Cut3 and Cut14 was necessary to reconstitute a reannealing activity in vitro (Sutani and Yanagida 1997). More quantitative analysis will be required to clarify this discrepancy. The amino-terminal domain of Smc2p (and Smclp), which contains the Walker A motif, binds to ATP but is apparently insufficient to hydrolyze it (Akhmedov et al. 1998).

Another important question concerns the target sequences of the condensin complex. Is it recruited to specific chromosomal sites to initiate condensation? 13S condensin is unlikely to display a strict sequence specificity and is more likely to recognize structural features of DNA instead, such as bent DNA or narrow minor grooves. It has been shown that the complex displays a high affinity for structured DNA, such as cruciform DNA (Kimura and Hirano 1997). Remarkably, a small fragment of the carboxy-terminal domain of Smc2p (and Smclp) appears to be able to recognize a cruciform DNA (Akhmedov et al. 1998). Future work must also address the question of whether, in the context of chromosomes, SMC proteins interact preferentially to scaffold-associated regions (SARs), cis-acting sequences implicated in higher-order chromosome dynamics (e.g., Strick and Laemmli 1995).

\section{Chromosomal targeting}

How does $13 \mathrm{~S}$ condensin initiate chromosome condensation specifically at the onset of mitosis? The cdc2-dependent activation of the supercoiling activity of $13 \mathrm{~S}$ condensin does not account for the whole mechanism. In cell-free extracts of Xenopus eggs, association of $13 \mathrm{~S}$ condensin with chromosomes is mitosis specific (Hirano et al. 1997). Nevertheless, the mitotic and interphase forms of $13 \mathrm{~S}$ condensin, once purified from the extracts, dis- 
play a comparable level of DNA-binding activity (Kimura et al. 1998). Therefore, an additional regulatory mechanism must exist at the level of chromosomal targeting. Three models can be considered. First, eukaryotic cells may have a mitosis-specific factor that actively participates in the loading of condensin on chromatin. Alternatively, an interphase-specific inhibitor would prevent $13 \mathrm{~S}$ condensin from binding to chromatin. Second, as the physiological target of $13 \mathrm{~S}$ condensin is likely to be chromatin rather than naked DNA, it is possible that the mitotic form of $13 \mathrm{~S}$ condensin has a higher affinity for nucleosomal DNA than the interphase form. Third, cell cycle-specific modification of nucleosomes may influence the interaction between chromatin and $13 \mathrm{~S}$ condensin. In this respect, mitosis-specific phosphorylation of histone $\mathrm{H} 3$ is of great interest. Recent experiments using a phosphoepitope-specific antibody demonstrated that there is a strong spatial and temporal correlation between phosphorylation of histone $\mathrm{H} 3$ at serine 10 and chromosome condensation (Hendzel et al. 1997). One attractive hypothesis is that mitotically phosphorylated histone $\mathrm{H} 3$ acts as a 'chromatin receptor' that recruits 13S condensin to chromosomes. At present, these three models are equally possible and yet not mutually exclusive. Clearly, multiple mechanisms must cooperate to ensure that chromosome condensation occurs only during mitosis in the eukaryotic cell cycle. An additional level of regulation might take place when condensation factors are transported into the nucleus (Matsusaka et al. 1998). Finally, it should be noted that cdc2 is unable to fully phosphorylate XCAP-H or XCAP-G in vitro (Kimura et al. 1998), suggesting that other mitotic kinases participate in condensin regulation.

\section{Relationship to chromosome-wide gene repression}

A functional link between mitotic chromosome condensation and chromosome-wide gene repression was first recognized when an SMC protein (DPY-27) was found to be an essential regulator of dosage compensation in Caenorhabditis elegans (Chuang et al. 1994). In this organism, hermaphrodites $(\mathrm{XX})$ reduce the level of transcripts from each of their $\mathrm{X}$ chromosomes to equalize X-linked gene expression with that of males (XO). An elegant combination of genetics and biochemistry has identified a protein complex containing DPY-27 that is specifically recruited to $\mathrm{X}$ chromosomes and executes dosage compensation (Chuang et al. 1996). The complex shows striking structural similarities to the Xenopus $13 \mathrm{~S}$ condensin complex: like $13 \mathrm{~S}$ condensin, the dosage compensation complex consists of two SMC subunits (DPY-27 and MIX-1) and two (or more) non-SMC subunits (Fig. 1; Table 1). MIX-1 is an SMC2-type protein that is required for both mitosis and dosage compensation (Lieb et al. 1998), whereas DPY-27 is an 'SMC4-variant'-type protein that is essential only for dosage compensation (Chuang et al. 1994). MIX-1 is believed to fulfill its mitotic functions through association with a more conventional SMC4-type protein that is distinct from DPY-27. DPY-26, one of the non-SMC subunits, shares a limited similarity to XCAP-H (Lieb et al. 1996; Hirano et al. 1997). More recently, it has been shown that DPY-28 is also a component of the complex and is homologous to XCAP-D2 (B. Meyer, pers. comm.). The dosage compensation complex is likely to regulate gene expression by altering the higher-order structure of $\mathrm{X}$ chromosomes, possibly through a mechanism similar to the one underlying chromosome condensation. Furthermore, these studies provide an evolutionarily remarkable example of how an SMC protein (MIX-1) can flexibly, yet selectively, change its SMC partner and adapt itself to participate in a different cellular process.

\section{Cohesins and sister-chromatid cohesion}

\section{Components}

Perhaps one of the least understood issues in eukaryotic chromosome dynamics is the mechanism of sister-chromatid cohesion. How is the linkage between sister chromatids established, maintained, and dissolved during the cell cycle? Recent studies have shown that a subclass of SMC proteins plays a key role in this important process. Smclp in S. cerevisiae, the founding member of the SMC family, was originally identified through a genetic screen for mutants that exhibit a high rate of minichromosome loss (Larionov et al. 1985) and was subsequently shown to be essential for proper segregation of natural chromosomes (Strunnikov et al. 1993). However, the specific role of Smc1p and Smc3p in segregation was not revealed until a new genetic screen was designed for mutants that show premature separation of sister chromatids (Michaelis et al. 1997). The screen identified four gene products required for sister-chromatid cohesion in mitosis, namely, Smc1p, Smc3p, Scc1p, and Scc2p. Scc1p (also called Mcd1p) was identified independently in a genetic screen for mutants that exhibit enhanced inviability after a mitotic arrest, as well as in a high-copy suppressor screen for an smc1 mutant (Guacci et al. 1997). Scc1p/ Mcd1p is homologous to $S$. pombe Rad21 implicated in DNA repair and mitosis (Birkenbihl and Subramani 1992; Birkenbihl and Subramani 1995; Tatebayashi et al. 1998) and is highly conserved among eukaryotic organisms, including humans (McKay et al. 1996). These results suggest the existence of an evolutionarily conserved machinery for sister-chromatid cohesion that may include homologs of these gene products.

This idea has been substantiated very recently by a biochemical study that identified protein complexes (cohesins) containing SMC1- and SMC3-type proteins from Xenopus egg extracts (Losada et al. 1998). Like condensins, Xenopus egg extracts contain two forms of the cohesin complexes with sedimentation coefficients of $9 \mathrm{~S}$ and 14S. The 9S form (9S cohesin) is a heterodimer of XSMC1 (SMC1-type) and XSMC3 (SMC3-type), whereas the $14 \mathrm{~S}$ form (14S cohesin) contains at least three additional subunits, p155, p120, and p95 (Fig. 1; Table 2). p120 is identical to XRAD21, the Xenopus homolog of yeast $\operatorname{Rad} 21 / \operatorname{Scc} 1 \mathrm{p} /$ Mcd1p. Although the identities of p155 and p95 are not known, they are immunologically 
distinct from the subunits of $13 \mathrm{~S}$ condensin, indicating that the two SMC protein complexes share no common subunits. Nevertheless, it should be noted that $14 \mathrm{~S}$ cohesin and $13 \mathrm{~S}$ condensin share striking similarities in their structural organization (Fig. 1). It will be of great interest to test whether $14 \mathrm{~S}$ cohesin has biochemical activities similar to those associated with $13 \mathrm{~S}$ condensin (Kimura and Hirano 1997). In the Xenopus egg cell-free extracts, 14S cohesin binds to interphase chromatin coincidentally with DNA replication, and most of the complexes dissociate from chromatin at the onset of mitosis. Immunodepletion of cohesins during interphase causes defects in sister chromatid cohesion in subsequent mitosis (Losada et al. 1998).

Different sets of genetic studies have identified proteins that potentially interact with the cohesin subunits. In $S$. cerevisiae, Smclp interacts genetically and physically with Trf4p (Castaño et al. 1996), a gene product originally found in a screen for mutants that become lethal in combination with a topoisomerase I mutation (Sadoff et al. 1995). Recent evidence suggests that a trf4 mutant also exhibits a cohesion defect (M. Christman, pers. comm.). In Aspergillus nidulans, SudA (SMC3-type protein) interacts genetically with BimD (Holt and May 1996) and SudD (Anaya et al. 1998), both of which are required for proper segregation of chromosomes. It remains to be determined whether any of these proteins are components of the $14 S$ cohesin complex. In $S$. pombe, Mis4, which is required for sister-chromatid cohesion, has been found to be homologous to $S$. cerevisiae Scc2p (Furuya et al. 1998). Immunoprecipitation experiments suggest that this new class of cohesion proteins (Mis4/Scc2p) functions in a complex that is distinct from cohesins.

\section{Establishment, maintenance, and dissolution of cohesion}

A recent study in yeast provides insight into how cohesin might establish the linkage between sister chromatids (Uhlmann and Nasmyth 1998). In yeast, Scclp/ Mcdlp is an unstable protein that is synthesized in late $\mathrm{G}_{1}$ and is degraded after the metaphase-anaphase transition. Using a system in which expression of Scclp/ Mcdlp during the cell cycle can be manipulated precisely, it has been shown that Scclp/Mcdlp's function becomes essential during $S$ phase when it is recruited to chromosomes. When Scclp/Mcdlp is expressed in $G_{2}$ phase, it still binds to chromosomes but fails to function properly. These results suggest that Scclp/Mcdlp can establish cohesion only when it binds to duplicating chromatin during $S$ phase. It is attractive to speculate that the establishment of sister-chromatid cohesion is directly coupled to passage of a replication fork, although no evidence for this hypothesis is currently available.

How is the cohesin-mediated cohesion dissolved when sister chromatids separate? In S. cerevisiae, loss of cohesion at the metaphase-anaphase transition is accompanied by Scc1p/Mcdlp's dissociation from chromatids (Michaelis et al. 1997). Recent evidence indicates that this process depends on Esplp, which exists in a complex with its inhibitor Pdslp before anaphase (Ciosk et al. 1998). It has been proposed that at the metaphase-anaphase transition, the anaphase-promoting complex (APC) triggers proteolysis of Pds1p, which in turn liberates Esplp's function that leads to the dissociation of Scc1/Mcd1p from sister chromatids. Although the dissociated Scclp/Mcdlp is subsequently degraded in an APC-dependent manner, functional consequence of this degradation is currently not known. In $S$. pombe, the Cut1-Cut2 complex, the homolog of Esp1p-Pds1p, may have an analogous role in sister-chromatid separation (Funabiki et al. 1996; Kumada et al. 1998).

In Xenopus, the $14 \mathrm{~S}$ cohesin complex associates with chromatin during $S$ phase, but most of the complex dissociates from it at the onset of mitosis (Losada et al. 1998). This behavior is different from that of Scclp/ Mcdlp in yeast, suggesting that sister-chromatid cohesion in higher eukaryotes might involve multilayered mechanisms. It has been proposed that a reorganization of cohesion occurs at the onset of mitosis when interphase cohesion is partially released and mitotic cohesion is reestablished (Fig. 2; Losada et al. 1998). The mitotic cohesion between condensed sister chromatids could be mediated by mitosis-specific factors that are distinct from cohesins. One such candidate is MEI-S332, a Drosophila protein that is recruited to the centromeres in prophase and displaced from them at the metaphaseanaphase transition (Moore et al. 1998). However, MEIS332's function is not essential in mitosis. This could be explained by the existence of redundant molecular mechanisms holding sister chromatids together at the centromeres. Alternatively, other proteins acting along the chromosome arms (e.g., the low level of cohesins left on mitotic chromosomes) would be sufficient to ensure cohesion at this stage. These studies also raise an important question of how the two different types of cohesion, cohesion along the chromosomal arms and cohesion at the centromeres, are differentially established and dissolved during mitotic cell cycle. The distinct functional roles of the two types of cohesion are more apparent in meiosis (Miyazaki and Orr-Weaver 1994). In S. cerevisiae, cohesins might mediate both arm cohesion and centromeric cohesion, and thereby act as the sole or predominant regulator of cohesion during the mitotic cycle.

\section{A link between cohesion and condensation?}

What is the functional relationship between sister-chromatid cohesion and condensation? Do cohesins and condensins interplay with each other? In yeast, mutations in a cohesin subunit (Scclp/Mcdlp) result in abnormal condensation (Guacci et al. 1997). This observation has led to a model proposing that Scclp/Mcd1p functions as a linker molecule that connects the cohesion and condensation machineries on mitotic chromosomes. In contrast, in Xenopus, there is no evidence for a requirement for the Scclp/Mcd1p homolog (XRAD21) in condensation (Losada et al. 1998). XRAD21 is a subunit of the cohesion machinery (14S cohesin) and does not associate 
Figure 2. Vertebrate chromosome dynamics regulated by two SMC protein complexes. As DNA replication proceeds during $S$ phase $(\mathrm{S})$, cohesins (green rectangles) associate with duplicating chromatin, establishing interphase cohesion $\left(\mathrm{G}_{2}\right)$. In vertebrates, most cohesins dissociate from chromosomes at the onset of mitosis when condensins (pink circles) are targeted to chromosomes to drive condensation $\left(\mathrm{G}_{2}\right.$ to metaphase). The low amount of cohesins left on the mitotic chromosomes might be sufficient to hold sister chromatids together until the metaphase-to-anaphase

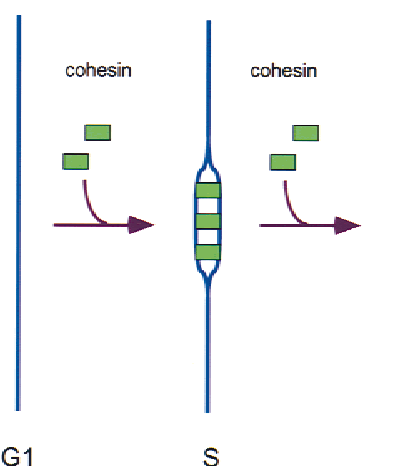

G1

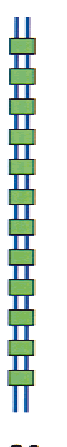

G2

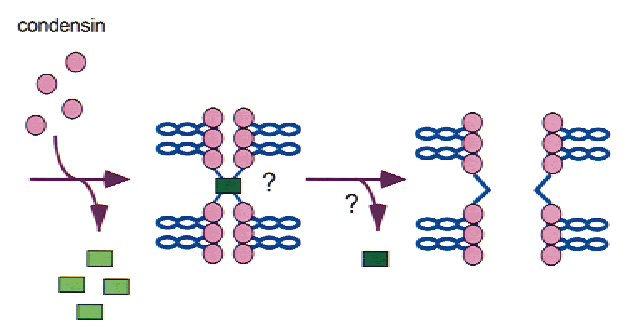

Metaphase

Anaphase

tors (e.g., MEI-S332) are activated or recruited to the chromosomes in prometaphase and support the linkage between mitotic chromatids. At the onset of anaphase, the mitotic 'glue' dissociates from chromosomes, allowing the two sister chromatids to separate. The single cohesion site drawn on the metaphase chromosome by no means implies that cohesion at this stage is restricted to the centromeric region. In yeast, cohesins do not dissociate from chromosomes at the onset of mitosis, and cohesin-mediated cohesion persists until the metaphase-anaphase transition.

with the condensation machinery (13S condensin). In the cohesin-depleted (thereby XRAD21-depleted) extracts, $13 \mathrm{~S}$ condensin can associate normally with chromosomes and drive their condensation. Thus, cohesion and condensation are largely separable in the Xenopus cellfree system (Fig. 2). The apparent discrepancy between the two systems may be explained by differential contribution of the condensation and cohesion machineries to metaphase chromosome architecture (Losada et al. 1998). Nevertheless, the structural similarity between cohesins and condensins suggests strongly that cohesion and condensation might have evolved from a common molecular mechanism. In the primitive chromosome cycle of bacteria, the two processes could be regulated by a single SMC protein (Hirano and Hirano 1998; Lin and Grossman 1998).

\section{Relationship to recombinational repair}

The identification of the two different SMC2-SMC4 complexes has provided a hitherto unexpected link between dosage compensation and mitotic chromosome condensation. Likewise, biochemical characterization of recombination complex-1 (RC-1) has uncovered a potential connection between sister-chromatid cohesion and recombinational repair. RC-1 was originally purified from calf thymus as a protein complex that catalyzes a recombinational repair reaction in vitro (Jessberger et al. 1993) and subsequently found to contain two SMC subunits (bSMC1 and bSMC3) in addition to DNA polymerase $\epsilon$ and ligase III (Fig. 1; Table 2; Jessberger et al. 1996). Although RC-1 and 14S cohesin share the SMC1- and SMC3-type subunits, the two complexes contain different sets of non-SMC subunits and are thereby distinct. The identification of Rad21 homologs as components of the cohesin complexes further emphasizes the link between cohesion and double-strand break repair (Guacci et al. 1997; Michaelis et al. 1997; Losada et al. 1998). Future studies should elucidate how SMC proteins ma- nipulate the interaction between sister DNA molecules and how they contribute to maintaining the genetic integrity in the interphase nucleus.

\section{SMC and bacterial chromosome mechanics}

A remarkable feature of SMC proteins is that they are conserved not only among eukaryotes but also in bacteria and archaea. The ancient origin of SMC proteins is likely to reflect their fundamental function in DNA dynamics. Whereas multiple SMC members have been found within single eukaryotic species, each of the bacterial or archaeal genomes fully sequenced so far contains either a single or no smc gene. Recently, two groups have reported the first genetic studies of the smc gene from the Gram-positive bacterium B. subtilis (Britton et al. 1998; Moriya et al. 1998). Null mutations in smc cause multiple phenotypes, including accumulation of anucleate cells, disruption of nucleoid structure, and misassembly of a protein complex involved in chromosome partitioning. It has been proposed that the B. subtilis $s m c$ gene product (BsSMC) contributes directly to the compaction of the nucleoid mass and that defects in this process result in the multiple phenotypes observed in the $s m c$ mutants. An immunolocalization study shows that BsSMC localizes to the chromosomes but is also present in discrete foci at polar positions in the cell (Graumann et al. 1998). Cell cycle-dependent structural changes of the polar foci suggest a dynamic movement of BsSMC within the cell and its functional contribution to chromosome condensation and segregation.

A biochemical analysis has demonstrated that BsSMC can function as a simple homodimer without any associated subunits (Hirano and Hirano 1998). Surprisingly, BsSMC binds preferentially to single-stranded DNA (ssDNA). It has a DNA-stimulated ATPase activity and the stimulation is much greater with ssDNA than with double-stranded DNA (dsDNA). Moreover, BsSMC can form large nucleoprotein aggregates in an ATP-depen- 
dent and ssDNA-specific manner. Although the in vitro evidence for dynamic interactions between BsSMC and ssDNA is strong, it remains to be determined whether the interactions are physiologically relevant. The energy-dependent aggregation of ssDNA might represent a primitive type of chromosome condensation that occurs during segregation of bacterial chromosomes. A role of ssDNA in chromosome condensation has also been suggested in S. pombe (Sutani and Yanagida 1997).

\section{SMC as an ATP-modulated DNA cross-linker}

One of the unique features of SMC proteins is that they have two long coiled-coil domains separated by a nonhelical hinge. A remarkable observation from a recent electron microscopic study is that the coiled-coils of the BsSMC homodimer are arranged into an antiparallel fashion (Melby et al. 1998). Such long antiparallel coiledcoil interactions ( 300 amino acids long) are unusual and have no precedent. The antiparallel arrangement, however, explains how two conserved ATP-binding motifs located in the amino- and carboxy-terminal domains (the Walker A and B motifs, respectively) can make direct contact with each other, constituting an ATP-binding pocket (Saitoh et al. 1994). Therefore, BsSMC has a symmetrical structure with two identical functional domains at the ends of the long arms (Fig. 3A). Because each end has the ability to interact with both ATP and DNA (Akhmedov et al. 1998), the SMC homodimer could be considered as an ATP-modulated DNA crosslinker. The structural study shows further that the central hinge is structurally flexible, allowing a 'scissoring' action of the SMC cross-linker. This novel mode of action might illustrate how BsSMC induces an aggregation of ssDNA (Hirano and Hirano 1998). It will be very important to understand how ATP-binding and hydrolysis modulate SMC functions, although it has been shown that DNA-binding per se does not require ATP (Hirano and Hirano 1998). Whatever the mechanism might be, it is no longer appropriate to emphasize the structural similarity between SMC proteins and cytoskeletal motor proteins because the latter proteins have a polar structure with the ATP-utilizing domains located at one end of the molecule.

Can the antiparallel mode of association be extended to eukaryotic SMC heterodimers? Although it remains to be tested experimentally, the answer is likely to be yes. Then the two classes of eukaryotic SMC complexes would be categorized into different types of DNA crosslinkers. The SMC2-SMC4-type complexes, which participate in chromosome condensation and dosage compensation, may be considered as an intramolecular DNA cross-linker that promotes compaction of a single DNA molecule (Fig. 3B, left). In contrast, the SMC1-SMC3type complexes, which are involved in sister chromatid cohesion and recombinational repair, can be regarded as an intermolecular DNA cross-linker that brings and holds two different DNA molecules together (Fig. 3B, right). Although this is obviously an oversimplified view, the cross-linker model provides us with a basic

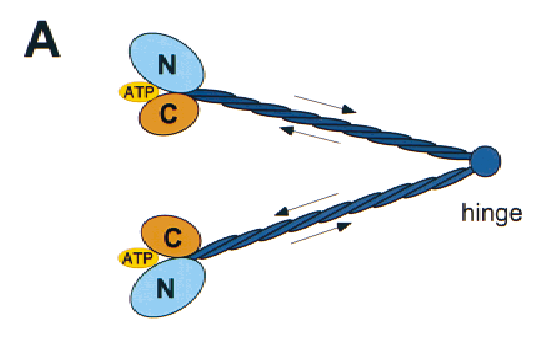

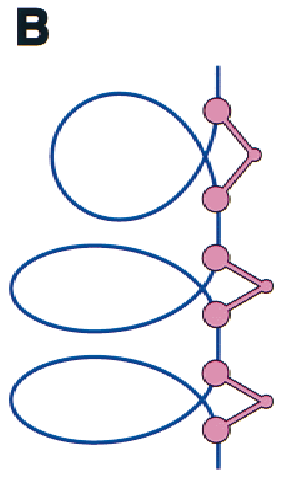

SMC2-SMC4

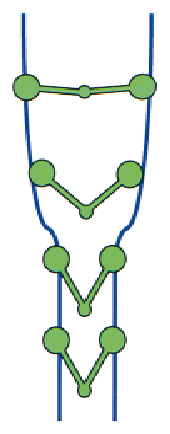

SMC1-SMC3
Figure 3. SMC proteins as an ATP-modulated DNA crosslinker. (A) A model of the BsSMC structure. The amino-terminal $(\mathrm{N})$ and carboxy-terminal (C) domains of BsSMC contain the Walker A and B motifs, respectively. The antiparallel coiledcoil interaction of two SMC polypeptides brings the two motifs together, constituting an ATP-binding site (ATP) at each end of the molecule. The flexible hinge region allows BsSMC to make a scissoring action. Arrows indicate the $\mathrm{N} \rightarrow \mathrm{C}$ direction of the polypeptide (adapted from Melby et al. 1998). (B) Hypothetical interactions of SMC protein complexes and DNA. The SMC2SMC4 (left) and SMC1-SMC3 (right) complexes may act as intramolecular and intermolecular DNA cross-linkers, respectively. For simplicity, the two SMC complexes are drawn as symmetrical structures with a flexible hinge.

framework for our understanding of how eukaryotic SMC protein complexes might work. In this scenario, we need to understand how the two classes of SMC protein complexes might distinguish between the intramolecular and intermolecular DNA interactions. Introduction of superhelical tension into DNA is likely to be one of the key mechanisms underlying the action of the SMC2SMC4-type complexes because superhelical tension propagates only intramolecularly (Kimura and Hirano 1997). One possible mechanism would be that the SMC2-SMC4 cross-linker mediates the formation of a specific type of DNA loops (e.g., loops with positive chirality) and thereby generates positive superhelical tension into DNA. Finally, it is important to note that the two ends of a eukaryotic SMC heterodimer are similar but not identical and therefore must have differentiated functional roles.

\section{Concluding remarks and future directions}

SMC proteins are key components that regulate a wide variety of chromosomal events from bacteria to humans. 
Although recent studies have uncovered diverse cellular functions and unique biochemical activities of this new class of chromosomal ATPases, we still do not understand how they are functionally related and mechanistically linked. In this paper a hypothesis is offered that ATP-modulated cross-linking of DNA represents the key mechanism underlying all actions of SMC dimers. In eukaryotes, combinatorial association of different SMC and non-SMC subunits provides an opportunity for each SMC complex to acquire a unique and more sophisticated activity required for its specialized cellular function. Future biochemical, genetic, and structural characterization of SMC proteins in the bacterial and eukaryotic systems should refine and extend this idea. It is also anticipated that a better understanding of SMC proteins will provide fundamental insights into the evolution of large-scale chromosome mechanics. Finally, it should be added that the potential roles of SMC protein complexes remain to be determined in a number of important areas, such as interphase nuclear architecture (e.g., heterochromatin formation) and meiotic chromosome dynamics and recombination.

\section{Acknowledgments}

I thank C. Wu, I. Ouspenski, H. Bellen, M. Christman, B. Meyer, and M. Yanagida for allowing me to cite their results prior to publication. I am also grateful to K. Nasmyth, R. Jessberger, A. Strunnikov, K. Yokomori, and M. Yanagida for preprints. This work was supported by grants from the National Institutes of Health, the Pew Scholars Program in the Biomedical Sciences, and the Human Frontier Science Program.

\section{References}

Akhmedov, A.T., C. Frei, M. Tsai-Pflugfelder, B. Kemper, S.M. Gasser, and R. Jessberger. 1998. Structural maintenance of chromosomes protein C-terminal domains bind preferentially to DNA with secondary structure. I. Biol. Chem. 273: 24088-24094.

Anaya, P., S.C. Evans, C. Dai, G. Lozano, and G.S. May. 1998. Isolation of the Aspergillus nidulans sudD gene and its human homologue. Gene 211: 323-329.

Bhat, M.A., A.V. Philp, D.M. Glover, and H.J. Bellen. 1996. Chromatid segregation at anaphase requires the barren product, a novel chromosome associated protein that interacts with topoisomerase II. Cell 87: 1103-1114.

Birkenbihl, R.P. and S. Subramani. 1992. Cloning and characterization of rad21, an essential gene of Schizosaccharomyces pombe involved in DNA double-strand-break repair. Nucleic Acids Res. 20: 6605-6611.

- 1995. The rad21 gene product of Schizosaccharomyces pombe is a nuclear, cell cycle-regulated phosphoprotein. $J$. Biol. Chem. 270: 7703-7711.

Britton, R.A., D.C.-H. Lin, and A.D. Grossman. 1998. Characterization of a prokaryotic SMC protein involved in chromosome partitioning. Gens \& Dev. 12: 1254-1259.

Cabello, O.A., A. Baldini, M. Bhat, H. Bellen, and J.W. Belmont. 1997. Localization of $B R R N 1$, the human homologue of Drosophila barr, to 2q11.2. Genomics 46: 311-313.

Castaño, I.B., P.M. Brzoska, B.U. Sadoff, H. Chen, and M.F. Christman. 1996. Mitotic chromosome condensation in the
rDNA requires TRF4 and DNA topoisomerase I in Saccharomyces cerevisiae. Genes \& Dev. 10: 2564-2576.

Chuang, P.-T., D.G. Albertson, and B.J. Meyer. 1994. DPY-27: A chromosome condensation protein homolog that regulates C. elegans dosage compensation through association with the X chromosome. Cell 79: 459-474.

Chuang. P.-T., J.D. Lieb, and B.J. Meyer. 1996. Sex-specific assembly of a dosage compensation complex on the nematode X chromosome. Science 274: 1736-1739.

Ciosk, R., W. Zachariae, C. Michaelis, A. Shevchenko, M. Mann, and K. Nasmyth. 1998. An ESP1/PDS1 complex regulates loss of sister chromatid cohesion at the metaphase to anaphase transition in yeast. Cell 93: 1067-1076.

Funabiki, H., K. Kumada, and M. Yanagida. 1996. Fission yeast Cut 1 and Cut 2 are essential for sister chromatid separation, concentrate along the metaphase spindle and form large complexes. EMBO J. 15: 6617-6628.

Furuya, K., K. Takahashi, and M. Yanagida. 1998. Faithful anaphase is ensured by Mis4, a sister chromatid cohesion molecule required in the $S$ phase and not destroyed in the $G_{1}$ phase. Genes \& Dev. 12: 3408-3418.

Graumann, P.L., R. Losick, and A.V. Strunnikov. 1998. Subcellular localization of Bacillus subtilis SMC, a protein involved in chromosome condensation and segregation. J. Bacteriol. 180: 5749-5755.

Guacci, V., D. Koshland, and A. Strunnikov. 1997. A direct link between sister chromatid cohesion and chromosome condensation revealed through the analysis of MCD1 in S. cerevisiae. Cell 91: 47-57.

Hendzel, M.J., Y. Wei, M.A. Mancini, A. Van Hooser, T. Ranalli, B.R. Brinkley, D.P. Bazett-Jones, and C.D. Allis. 1997. Mitosis-specific phosphorylation of histone $\mathrm{H} 3$ initiates primarily within pericentromeric heterochromatin during $G_{2}$ and spreads in an ordered fashion coincident with mitotic chromosome condensation. Chromosoma 106: 348-360.

Hirano, T. 1998. SMC protein complexes and higher-order chromosome dynamics. Curr. Opin. Cell Biol. 10: 317-322.

Hirano, M. and T. Hirano. 1998. ATP-dependent aggregation of single-stranded DNA by a bacterial SMC homodimer. EMBO I. 17: 7139-7148.

Hirano, T. and T.J. Mitchison. 1994. A heterodimeric coiled-coil protein required for mitotic chromosome condensation in vitro. Cell 79: 449-458.

Hirano, T., R. Kobayashi, and M. Hirano. 1997. Condensins, chromosome condensation protein complexes containing XCAP-C, XCAP-E and a Xenopus homolog of the Drosophila Barren protein. Cell 89: 511-521.

Holt, C.L. and G.S. May. 1996. An extragenic suppressor of the mitosis-defective bimD6 mutation of Aspergillus nidulans codes for a chromosome scaffold protein. Genetics 142: 777787.

Hong, C.-S. and B. Ganetzky. 1996. Molecular characterization of neurally expressing genes in the para sodium channel gene cluster of Drosophila. Genetics 142: 879-892.

Jessberger, R., V. Podust, U. Hubscher, and P. Berg. 1993. A mammalian protein complex that repairs double-strand breaks and deletions by recombination. J. Biol. Chem. 268: 15070-15079.

Jessberger, R., B. Riwar, H. Baechtold, and A.T. Akhmedov. 1996. SMC proteins constitute two subunits of the mammalian recombination complex RC-1. EMBO J. 15: 4061-4068.

Jessberger, R., C. Frei, and S.M. Gasser. 1998. Chromosome dynamics: The SMC protein family. Curr. Opin. Genet. Dev. 8: 254-259.

Kimura, K. and T. Hirano. 1997. ATP-dependent positive supercoiling of DNA by 13 S condensin: A biochemical implica- 
tion for chromosome condensation. Cell 90: 625-634.

Kimura, K., M. Hirano, R. Kobayashi, and T. Hirano. 1998. Phosphorylation and activation of $13 \mathrm{~S}$ condensin by cdc 2 in vitro. Science 282: 487-490.

Koshland, D. and A. Strunnikov. 1996. Mitotic chromosome condensation. Annu. Rev. Cell Dev. Biol. 12: 305-333.

Kumada, K., T. Nakamura, K. Nagao, H. Funabiki, T. Nakagawa, and M. Yanagida. 1998. Cutl is loaded onto the spindle by binding to Cut2 and promotes anaphase spindle movement upon Cut2 proteolysis. Curr. Biol. 8: 633-641.

Larionov, V., T. Karpova, N. Kouprina, and G. Jouravleva. 1985. A mutant of Saccharomyces cerevisiae with impaired maintenance of centromeric plasmids. Curr. Genet. 10: 15-20.

Lieb, J.D., E.E. Capowski, P. Meneely, and B.J. Meyer. 1996. DPY-26, a link between dosage compensation and meiotic chromosome segregation in the nematode. Science 274: 1732-1736.

Lieb, J.D., M.R. Albrecht, P.-T. Chuang, and B.J. Meyer. 1998. MIX-1: An essential component of the C. elegans mitotic machinery executes X-chromosome dosage compensation. Cell 92: 265-277.

Lin, D.C.-H. and A.D. Grossman. 1998. Identification and characterization of a bacterial chromosome partitioning site. Cell 92: 675-685.

Losada, A., M. Hirano, and T. Hirano. 1998. Identification of Xenopus SMC protein complexes required for sister chromatid cohesion. Genes \& Dev. 12: 1986-1997.

Matsusaka, T., N. Imamoto, Y. Yoneda, and M. Yanagida. 1998. Muations in fission yeast Cut15, an importin homolog, lead to mitotic progression without chromosome condensation. Curr. Biol. 8: 1031-1034.

McKay, M.J., C. Troelstra, P. van der Spek, R. Kanaar, B. Smit, A. Hagemeijer, D. Bootsma, and J.H.J. Hoeijmakers. 1996. Sequence conservation of the rad21 Schizosaccharomyces pombe DNA double-strand break repir gene in human and mouse. Genomics 36: 305-315.

Melby, T.E.G., C.N. Ciampaglio, G. Briscoe, and H.P. Erickson. 1998. The symmetrical structure of structural maintenance of chromosomes (SMC) and MukB proteins: Long, anitparallel coiled coils, folded at a flexible hinge. I. Cell Biol. 142: 1595-1604.

Michaelis, C., R. Ciosk, and K. Nasmyth. 1997. Cohesins: Chromosomal proteins that prevent premature seperation of sister chromatids. Cell 91: 35-45.

Miyazaki, W.Y. and T.L. Orr-Weaver. 1994. Sister-chromatid cohesion in mitosis and meiosis. Annu. Rev. Genet. 28: 167187.

Moore, D.P., A.W. Page, T.T.L. Tang, A.W. Kerrebrock, and T.L. Orr-Weaver. 1998. The cohesion protein MEI-S332 localizes to condensed meiotic and mitotic centromeres until sister chromatids separate. J. Cell Biol. 140: 1003-1012.

Moriya, S., E. Tsujikawa, A.K. Hassan, K. Asai, T. Kodama, and N. Ogasawara. 1998. A Bacillus subtilis gene-encoding protein homologous to eukaryotic SMC motor protein is necessary for chromosome partition and condensation. Mol. Microbiol. 29: 179-187.

Rocques, P.J., J. Clark, S. Ball, J. Crew, S. Gill, Z. Christodoulou, R.H. Borts, E.J. Louis, K. Davies, and C.S. Cooper. 1995. The human SB1.8 gene (DXS423) encodes a putative chromosome segregation protein conserved in lower eukaryotes and prokaryotes. Mol. Hum. Genet. 4: 243-249.

Sadoff, B.U., S. Heath-Pagliuso, I.B. Castaño, Y. Zhu, F.S. Kieff, and M.F. Christman. 1995. Isolation of mutants of Saccharomyces cerevisiae requiring DNA topoisomerase I. Genetics 141: 465-479.

Saitoh, N., I. Goldberg, E.R. Wood, and W.C. Earnshaw. 1994.
ScII: An abundant chromosome scaffold protein is a member of a family of putative ATPases with an unusual predicted tertiary structure. J. Cell Biol. 127: 303-318.

Saka, Y., T. Sutani, Y. Yamashita, S. Saitoh, M. Takeuchi, Y. Nakaseko, and M. Yanagida. 1994. Fission yeast cut3 and cut14, members of a ubiquitous protein family, are required for chromosome condensation and segregation in mitosis. EMBO J. 13: 4938-4952.

Schmiesing, J.A., A.R. Ball, H.C. Gregson, J.M. Alderton, S. Zhou, and K. Yokomori. 1998. Identification of two distinct human SMC protien complexes involved in mitotic chromosome dynamics. Proc. Nat1. Acad. Sci. 95: 12906-12911.

Shimizu, K., H. Shirataki, T. Honda, S. Minami, and Y. Takai. 1998. Complex formation of SMAP, a KAP3A/B ATPase motor-associated protein, with a human chromosome-associated polypeptide. J. Biol. Chem. 273: 6591-6594.

Strick, R. and U.K. Laemmli. 1995. SARs are cis DNA elements of chromosome dynamics: Synthesis of a SAR repressor protein. Cell 83: 1137-1148.

Strunnikov, A.V., V.L. Larionov, and D. Koshland. 1993. SMC1: An essential yeast gene encoding a putative head-rod-tail protein is required for nuclear division and defines a new ubiquitous family. J. Cell Biol. 123: 1635-1648.

Strunnikov, A.V., E. Hogan, and D. Koshland. 1995. SMC2, a Saccharomyces cerevisiae gene essential for chromosome segregation and condensation, defines a subgroup within the SMC family. Genes \& Dev. 9: 587-599.

Sutani, T. and M. Yanagida. 1997. DNA renaturation activity of the SMC complex implicated in chromosome condensation. Nature 388: 798-801.

Tatebayashi, K., J. Kato, and H. Ikeda. 1998. Isolation of a Schizosaccharomyces pombe rad $21^{\text {ts }}$ mutant that is aberrant in chromosome segregation, microtubule function, DNA repair and sensitive to hyrdoxyurea: Involvement of Rad21 in ubiquitin-mediated proteolysis. Genetics 148: 4957.

Uhlmann, F. and K. Nasmyth. 1998. Cohesion between sister chromatids must be established during DNA replication. Curr. Biol. 8: 1095-1101.

Yanagida, M. 1998. Fission yeast cut mutations revisited: Control of anaphase. Trends Cell Biol. 8: 144-149. 


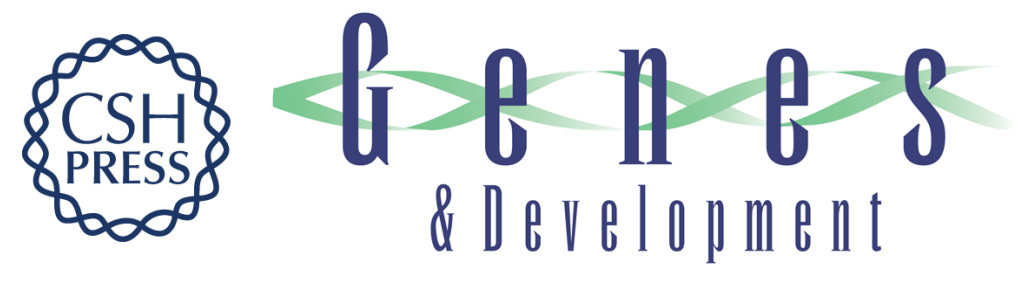

\section{SMC-mediated chromosome mechanics: a conserved scheme from bacteria to vertebrates?}

Tatsuya Hirano

Genes Dev. 1999, 13:

Access the most recent version at doi:10.1101/gad.13.1.11

$\begin{array}{ll}\text { References } & \text { This article cites } 54 \text { articles, } 23 \text { of which can be accessed free at: } \\ \text { http://genesdev.cshlp.org/content/13/1/11.full.html\#ref-list-1 }\end{array}$

License

Email Alerting Receive free email alerts when new articles cite this article - sign up in the box at the top Service right corner of the article or click here.

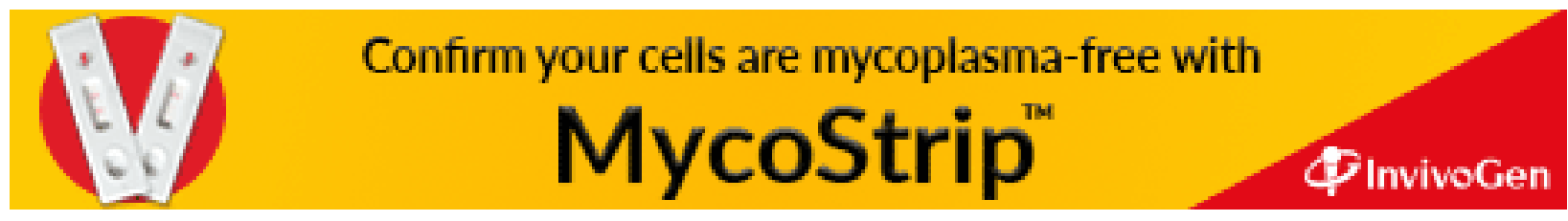

